\title{
Portable Document Format
}

National Cancer Institute

\section{Source}

National Cancer Institute. Portable Document Format. NCI Thesaurus. Code C63805.

An open file format created and controlled by Adobe Systems, for representing two-

dimensional documents in a device independent and resolution independent fixed-layout document format. Each PDF file encapsulates a complete description of a 2D document that includes the text, fonts, images, and 2D vector graphics that compose the document. PDF files do not encode information that is specific to the application software, hardware, or operating system used to create or view the document. This feature ensures that a valid PDF will render exactly the same regardless of its orig in or destination (but depending on font availability when fonts are not encapsulated in the file). 